\title{
Lo público y lo privado como problema prepolítico. Un análisis desde la sociología de la comunicación
}

\author{
Luciano H. Elizalde \\ Profesor de Comunicación Política. Universidad Austral (Argentina)
}

Resumen:

El objetivo del trabajo es presentar una hipótesis alternativa para explicar el origen social de la diferenciación entre lo público y lo privado. La metodología usada fue hipotética deductiva ya que se puso en discusión los puntos de vista teórico, político y sociológico, de la diferencia entre lo público y lo privado, para luego generar una conjetura que integrara y diera un significado diferente a las posiciones establecidas por Norberto Bobbio, Jürgen Habermas, Norbert Elias y Anthony Giddens. El resultado que se ha obtenido es una teoría con un grado operativo más importante en la medida en que es posible "medir" la transformación entre lo público y lo privado por medio de la formación de los agentes sociales (agentivación) y de los actores sociales (actoralización).

Palabras clave:

Escenario público, medios de comunicación, público-privado

Abstract:

The objective of this work is to present an alternative hypothesis to explain the origin of social differentiation between public and private. The methodology used was hypothetical and deductive which was under discussion viewpoints theoretical, political and sociological, of the difference between public and private, and then generate a conjecture that integrate and give a different meaning to the positions established by Norberto Bobbio, Jürgen Habermas, Norbert Elias and Anthony Giddens. The result has been obtained is a theory operating with a degree more important to the extent that it is possible to "measure" the transformation between the public and private sector through the formation of the social partners (agentivacion) and the social actors (actoralización).

Key words:

Public sphere, mass media, public-private 


\section{Introducción y planteamiento del problema}

El objetivo general de este trabajo es desarrollar una teoría sobre el origen social de la diferencia entre lo público y lo privado desde el punto de vista de la sociología de la comunicación pública colectiva. La sociología de la comunicación considera el escenario público como lugar, ámbito y contexto de acción y de discurso, por un lado, y la comunicación pública colectiva como proceso de cambio y de estabilización de los cambios, por otro; son categorías que cruzan estos problemas. Este problema, de alguna manera, es el que tiene en mientes Luis Núñez Ladevéze cuando se pregunta hasta dónde la diferencia entre lo privado y lo público es algo convencional o natural, en tanto necesita establecer los alcances y los límites entre una ética pública y una ética privada para alcanzar niveles de solidaridad social políticamente estabilizadores, (Núñez Ladevéze, 2000: 102 y ss.). En el marco de la sociedad postmoderna, dice Núñez Ladevéze (2000: 113) -que delimita el escenario público para que exista tolerancia — se pregunta: “¿qué tipo de acuerdo asegura un pacto ex post orientado a imponer political correctness para la que es socialmente equiparable la multivocidad de conductas privadas?". Desde una concepción filosófico política, considera que en la actualidad habría que responderse, además, a otra pregunta: “ipor qué obedecer una ética cuyo imperativo brota de la hipotética utilidad de un deber abstracto, que no surge de la adhesión ni la suscita, ajena a la identidad social y cuyos límites son, por definición, relativos, imprecisos y discutibles?” (Núñez Ladevéze, 2000: 116).

En realidad, traduciendo la filosofía política en sociología, las respuestas a estas preguntas podrían plantearse de la siguiente manera: primero, el "acuerdo" desaparece de la generalización social, es decir, no existe una funcionalización social del acuerdo social; en segundo lugar, el motivo de obedecer a una ética pública no se funda en el "acuerdo", sin embargo, como dice Núñez Ladevéze, es factible el funcionamiento de la coacción; pero una coacción que no puede identificársela sólo con el funcionamiento hobbesiano del Estado, sino también con un proceso de re-elaboración y de construcción de agentes y de actores sociales, que encuentran límites concretos para sus deseos, como también ampliación de sus horizontes en la medida en que aumenta su "soberanía" social y que tienen posibilidades, aunque no ideales, de aparecer y de ocultarse, de utilizar argumentos o de comunicar y proyectar su imagen o reputación para obtener crédito social.

Desde esta perspectiva de la diferencia entre lo público y privado, es posible analizar algunos problemas políticos, como son las cuestiones de representación, de gobernabilidad, de inclusión social o de legitimidad, como expresión y manifestación de las diferencias y de los cambios entre lo público y lo privado. La relación entre lo público y la comunicación colectiva surge en cada una de estas categorías: el proceso de representación en las democracias indirectas necesita de formas más eficaces de publicidad y de discusión -más allá del pesimismo schimittiano (Schmitt, 1990: 65 y ss.) — que, por otro lado, en las sociedades modernas 
se encuentran dentro de la relación que determina la prensa, el Parlamento y la opinión pública; sin embargo, como dice Luís Núñez Ladevéze (2000: 116), "es un insulto a la inteligencia insinuar siquiera que los centros de decisión política lo son de debate, de dialéctica o de argumentación. El procedimiento decisorio es meramente voluntarista, una emanación de la voluntad de poder, de la necesidad derivada de la falta de consenso para imponer una decisión a quien no quiere adoptarla"; esto significa que el escenario público no es guiado ni organizado por principios del modelo habermasiano burgués (Habermas, 1986: 17 y ss.); la cuestión de la gobernabilidad, como relación entre gobernados y gobernantes, es sin duda un tema que necesita ser planteado dentro de la estructura y de los principios de regulación del escenario público: hasta dónde los gobernados deben saber acerca de los gobernantes y cómo deberían hacer éstos para informar a los primeros; la inclusión social, por otro lado, que es mucho más que un problema de forma de representación discursiva de alguien en el escenario público, implica, de todos modos, tratar comunicativamente a los agentes sociales para que sean "incluidos" y que así pertenezcan al universo de sentido de la cultura política de una sociedad (Vasilachis de Giandino, 2003: 99 y ss.; 167 y ss.).

Finalmente, en el proceso de construcción de la legitimidad de un gobierno, la participación en el escenario público es una cuestión central de comunicación pública; el proceso político argentino, sobre todo considerado desde el año 2003, es un buen ejemplo: el presidente Kirchner consiguió aumentar su "legitimidad" -puesta en duda por haber ganado en segunda vuelta con un apoyo exiguo- por medio del consenso obtenido en el marco de sus acciones en la escena pública. Para Mario Riorda, la primera función de la comunicación gubernamental es la de mantener la legitimidad (Riorda, 2006: 33) y es factible de considerarla como un "mecanismo" de construcción de consenso (Elizalde, 2006: 180-184). Con esto no pretendo decir que sea la única manera de observar y de explicar las cuestiones de la representación, la gobernabilidad e inclusión social. Por el contrario, se podría decir que América Latina tiene cuestiones más urgentes, por lo menos a simple vista, que las aquí planteadas. Pero desde una concepción menos epidérmica, es poco realista pensar en un sistema político que dirija a la sociedad hacia una salida y no hacia otro atolladero, y al mismo tiempo, no reflexionar sobre una zona pública de vida que refleje la realidad por medio de una comunicación que distribuye colectivamente discursos sociales que cambian pacíficamente la sociedad.

Sin embargo, la cuestión planteada y el camino no andado no será el de la filosofía política, aunque se utilicen sus preguntas. El objetivo de este trabajo es plantear y esbozar una solución a un problema prepolítico que presenta consecuencias políticas. El problema que se tratará de resolver trata acerca del origen, de la formación y de las diferencias sociológicas de lo público y de lo privado. Esta es una cuestión central para varias disciplinas: en primer lugar, para la ciencia política, pero también para el derecho, para la sociología general y para la sociología de la comunicación. 
Un buen ejemplo de cómo es planteado y comúnmente definido el problema se encuentra en Norberto Bobbio (1989). Él dice que la "dicotomía público-privado" es originaria o constitutiva del campo de la teoría y de la filosofía política. Esto significa que son conceptos fundantes o que sirven de fundamento del resto de los que forman una teoría o una disciplina. La "gran dicotomía" de la teoría política es la diferencia entre lo público y lo privado, dice Bobbio (1989: 25 y ss.), en un intento por plantear las bases mínimas de una "teoría general de la política". A continuación explica que existen dos significados que han sido permanentes a lo largo de la historia de la filosofía política y de la teoría del derecho. Por un lado, se ha definido lo privado como aquello que se identifica con lo "individual" y lo público con lo "colectivo", y por otro, existe una teoría que considera lo privado como lo "secreto" y lo público como lo "no secreto".

En síntesis, Bobbio muestra magistralmente que la diferencia entre lo público y lo privado ha acompañado a Occidente durante toda la historia a la filosofía y a la teoría política. Y esta situación la presenta como una dicotomía constitutiva y originaria para una "teoría general de la política". El intento de Bobbio es absolutamente lógico. Desde el punto de vista de la ciencia política, es relevante plantear la diferencia entre lo que se considera y lo que no se considera una parte del campo de la política. La diferencia entre la vida pública y aquello que se coloca dentro de lo privado permite una división que delimita entre lo que es el campo mismo de la política y lo que no pertenece a su ámbito y que puede estar bajo el estudio de la economía, de la sociología, de la psicología o del derecho -específicamente, del derecho privado.

La perspectiva desde la cual la teoría de la comunicación y su rama más relacionada con la ciencia política -la sociología de los efectos de los medios de comunicación de masas- han observado y observan el problema de la distinción entre lo privado y lo público es diferente aunque complementario. La sociología de la comunicación de masas no se había interesado en el problema de las consecuencias que trae aparejada esta dicotomía hasta hace muy poco tiempo (Groselin, 1998; Heath, 1997; Lavigne, 1998; Thompson, 2001; Beuchamp, 1998; Burke, 1995). Además, los estudios de sociología de la comunicación de masas que se concentran en el problema del espacio público han permanecido en la aplicación de ciertos conceptos definidos por la filosofía, la teoría política, la sociología o el derecho. Aún hace falta, por lo menos de un modo teóricamente relevante, un planteo que considere la naturaleza de la relación entre lo social, lo público y la comunicación colectiva.

En definitiva, lo que se ha hecho para explicar el origen de lo público y de lo privado, sus transformaciones y sus consecuencias no ha sido demasiado. En primera instancia, lo que se puede encontrar como un lugar común es que las definiciones de lo privado y de lo público suelen ser definiciones y estudios de tipo sustancialistas (Glaser y Strauss, 1967: 134 y ss.). Es decir, son definiciones hechas con aquello que se considera 
el contenido de lo que se define como ámbito privado o ámbito público de vida. Un ejemplo relevante de esto es la manera en que Jürgen Habermas (1986) define el espacio público burgués. Comienza con la diferencia entre lo privado y lo público como categorías constitutivas, originarias, es decir, como si fueran el fundamento de otras categorías o realidades; por eso, estudia el desarrollo, las continuidades y discontinuidades del espacio público desde el punto de vista de su estructura y de sus principios de regulación. Lo público y lo privado son diferencias relacionadas con la ideología y la teoría o filosofía de las clases dirigentes y sus intereses para diferenciar la sociedad entre una cosa y otra. Finalmente, su conclusión se orienta a criticar el modo en que los cambios de estructura y de principios de regulación han producido patologías y deformaciones políticas en la sociedad del siglo XX. Por otro lado, en sus últimos trabajos sobre derecho y teoría política $(1998,1999)$, Habermas considera las posibilidades y los obstáculos que la estructura del espacio público actual incluye en el desarrollo de una democracia real y no meramente formal. En definitiva, Habermas está interesado en criticar una forma de espacio público y por delinear otra que considera especialmente beneficiosa para la democracia real y deliberativa que él propone (1999: 242 y ss.). Sin embargo, no aporta más que en los años sesenta al estudio de los cambios y transformaciones de lo público y de lo privado en las sociedades actuales.

Desde un punto de vista histórico sociológico, Norbert Elias (1998) estudió el espacio privado o el proceso de privatización -que es la contra-cara del proceso de publicitación- de tal modo que se acercó más a preguntarse sobre la manera en que las sociedades definen qué es privado y qué es público. Elias utilizó su teoría de la civilización para explicar de qué forma y por qué método se produce el proceso de privatización de las conductas sociales. En su estudio original sobre los procesos sociogenéticos y psicogenéticos en la sociedad de la Alta Edad Media europea, Elias mostró que los mecanismos civilizatorios de control y autocontrol definían las fronteras reales entre las nuevas y viejas formas de lo público y de lo privado. En este trabajo importantísimo para la teoría social y para las ciencias humanas, Elias se embarca en el estudio de los "medios" o de los "mecanismos" que llevaron a la formación de las diferencias entre lo público y lo privado, entre otros resultados que analiza. Pero no se pregunta acerca de qué es lo que se anticipa o permanece detrás de lo público y privado. Su aporte indiscutible y central a la teoría social fue mostrar de un modo tangible cómo funcionan los mecanismos civilizatorios. Y desde este punto de vista, estos mecanismos colaboran en la organización de las zonas de vida pública, por un lado, y de vida privada por otro.

Otra hipótesis que es necesario considerar para comprender de qué manera funcionan en la realidad social las diferencias de la vida privada y de la vida pública, es la de Anthony Giddens (1995b). Giddens afirma que las transformaciones que han sufrido las fronteras que dividen estas dos zonas de vida social son el re- 
sultado de un proceso de publicitación y de racionalización pública de la intimidad. Después de que la sociedad moderna, dice Giddens, instituyera la forma racional de comportarse en público como la única manera legítima para la vida social respetable y políticamente correcta, el método de discusión racional y de publicitación o desocultamiento de la acción se salió del marco de lo racional y pasó al de lo no racional. El método racional por medio del cual se debía hacer política, la manera de llegar a la "mayoría de edad" según Kant (1993) por medio de un uso público de la razón, fue trasladado para el uso y el tratamiento de otro referente que no era, precisamente, demasiado racional: la intimidad, el inconsciente, lo emocional, lo subjetivo. El efecto social que tuvo el conocimiento y la popularización de la psicoterapia, sobre todo del psicoanálisis en las sociedades del siglo $\mathrm{XX}$, fue fundamental para que se produjera este proceso de publicitación y racionalización de lo íntimo.

Ambos procesos, el de la publicitación de lo racional y el de la publicitación de lo íntimo, se transformaron en el marco y en el contexto en el que funcionan los géneros y los textos de la industria cultural. En otro lado he intentado explicar en qué medida estos procesos que caracterizan a la vida pública definen las características centrales de la comunicación pública colectiva en cualquier época histórica (Elizalde, 1998). Y si se tiene presente el modo en que la comunicación pública colectiva -tanto la orientada a la producción de la información de realidad como la que produce y distribuye ficción-influye en la vida política de una sociedad, se cierra el círculo de determinación: ciertas características de lo público determinan ciertas características de la comunicación pública colectiva que, a su vez, condiciona y define algunos rasgos culturales centrales de la política de una sociedad.

De acuerdo con estas hipótesis, la diferenciación entre lo público y lo privado desde la premodernidad hasta nuestros días se realizó por medio de un doble proceso: en primer lugar, durante los momentos previos e inmediatamente posteriores a la formación de la sociedad moderna (entre los siglos XV y XIX, aproximadamente) se publicitó todo aquello que se identificó y se definió como lo racional y como lo objetivamente social, o lo que afectaba a todas las partes de una sociedad; en segundo término y a lo largo de la mitad del siglo XIX y durante todo el siglo XX, se desarrolló un proceso social que, por medio del método de publicitación institucionalizado antes y aprendido por las personas sociales para gestionar lo racional, comenzó a aplicarse sobre el "material" emocional e íntimo de los seres humanos.

Hasta el siglo XIX, la sociedad del capitalismo liberal y de la democracia representativa publicitó lo racional y formó personas que ocultaban lo emocional (Elias, 1993; 1996); desde ese momento hasta finales del siglo XX, sin embargo, el mecanismo de publicitación y de racionalización ya no sólo sirvió para aquello que era en sí mismo algo racional, sino también para lo que se había considerado hasta entonces como in- 
timidad y realidad emocional: así se comenzó a mostrar, de un modo legítimo, lo íntimo y lo privado (Giddens, 1995b). En definitiva, los cambios que se perciben en la actualidad sobre aquello que es privado y aquello que es público dependen de estos dos procesos.

Sin embargo, estos mecanismos civilizatorios no alcanzan para explicar todo el problema. La identificación de estos mecanismos sociales y políticos de formación y de reformación de las fronteras entre lo público y lo privado no permiten resolver los problemas que se encuentran antes de las diferencias entre estas dos zonas de vida social. Aunque se cuente con hipótesis suficientemente explicativas de las condiciones y mecanismos de cambio de la vida privada y pública, aún no se ha expuesto una teoría que explique cuál es el origen fáctico de las distinciones entre la vida privada y la vida pública.

En resumen, la división entre lo privado y lo público de acuerdo con la distinción de Bobbio entre lo "individual" versus lo "colectivo", por un lado, y lo "secreto" versus lo "no secreto", por otro, es una diferencia constitutiva para la teoría política. Sin embargo, es necesario realizar una definición sociológica y pre-política para que se comprenda cuáles son sus consecuencias, tanto funcionales como disfuncionales, sobre los sistemas políticos. La definición de Bobbio parece ser acertada a los fines de desarrollar una teoría política general y amplia. Pero no aclara el origen conceptual, epistemológico, social e histórico de la división entre lo privado y lo público.

Sería necesario, entonces, identificar cuáles son los criterios subyacentes a la dicotomía privado público, qué es lo que hace que las sociedades humanas dividan la vida social en pública y privada, qué funciones cumple esta división y de qué manera afecta al desarrollo de la representación política, de la gobernabilidad y de la inclusión social. Sobre la base de las explicaciones y de sus lagunas, sería conveniente plantear los siguientes interrogantes:

(a) ¿Cuál es el origen sociológico que subyace a la dicotomía privado público? ¿Por qué y para qué las sociedades humanas definen zonas públicas y privadas de vida?

(b) ¿Qué criterios sociales son los que hacen o desarrollan esta diferencia entre las dos formas de sociabilidad?

(c) ¿Qué tipos de procesos sociales sirven de mecanismos para que la vida se divida en privada y pública?

(d) ¿De qué manera se desarrolla esto en las sociedades históricas? 


\section{Hipótesis}

La diferencia entre lo público y lo privado surge de dos procesos sociales básicos, definidos por la filosofía y por la teoría política como procesos prepolíticos: el proceso de autonomización o desautonomización social, por un lado, y el de ocultamiento o de exposición, por otro. Ambos procesos sociales, aunque se los considere prepolíticos, están permanentemente presentes a lo largo de la transformación y de la definición fáctica de lo que se denomina "espacio público" (Habermas) o "esfera pública" (Arendt). Y aun siendo procesos prepolíticos, afirmo que influyen de forma constante sobre la manera de definir y de ordenar la estructura y las pautas de comportamiento del espacio o esfera pública. La consecuencia de esto es que la definición y los cambios en la identidad de lo que se llama espacio público se encuentran en estos procesos sociales (prepolíticos) que se necesitan explicar.

A continuación se expondrán las proposiciones que conforman la hipótesis central de este trabajo:

Proposición 1. La diferencia entre la vida pública y la vida privada en una sociedad en un momento concreto de su historia está determinada por dos procesos sociales que se producen de modo constante: por un lado, un proceso de autonomización y desautonomización de los agentes sociales que actúan y se desempeñan en la sociedad, y por otro, un proceso de exposición y ocultamiento de ciertos tipos de comportamiento.

Proposición 2: Ambos procesos son el resultado de la determinación que producen las representaciones sociales de los metarrelatos en las formas institucionalizadas de vida.

Proposición 3: Los procesos de autonomización y de exposición-ocultamiento definen un orden social similar al de un escenario de actuación y su correspondiente zona de bastidores o bambalinas, que terminará por afectar y cambiar la autonomización y la exposición-ocultamiento.

Se planteará seguidamente el modo en que estos procesos de autonomización y de publicitación se activan hasta formar una estructura de escenario sobre el que actúan los agentes y condicionan la representación, la gobernabilidad y la inclusión social.

\section{Desarrollo teórico}

2.1. Las metanarrativas, por un lado, y las ideologías y las representaciones colectivas de las clases y de los grupos sociales dirigentes, por otro, determinan lo que es público y lo que es privado por medio de la definición fáctica de lo que tiene autonomía y de aquello que debería ser mostrado y lo que debería ser ocultado de la percepción de otros

$134 \mid n^{\circ} 7$ | doxa.comunicación 
El problema de los procesos de clasificación social (Durkheim, 1991; Fleck, 1986; Douglas, 1996) se podría dividir en dos niveles de acuerdo con su nivel de abstracción: un nivel de mayor abstracción definido por los metarrelatos (Lyotard, 1989), las metanarrativas (Somers, 1995: 229 y ss.) o las formaciones discursivas (Foucault, 1970) y un nivel menos abstracto de clasificación que se encuentra en las ideologías de los grupos dirigentes o con mayor poder relativo, compuestas por representaciones sistemáticamente desarrolladas e impuestas, por estructuras semánticas manifiestas y por conceptos definidos abiertamente (Van Dijk, 1995; Vasilachis de Gialdino, 1997).

En un primer nivel de más abstracción, se encuentran los metarrelatos o metanarrativas. Estos sistemas de conceptos, relatos y teorías definen de un modo muy general quiénes son las agencias humanas ideales. Las agencias humanas son los tipos de sujetos o agentes a los que se les asigna la función de comportarse de un modo, de tener cierta responsabilidad y responder a determinadas obligaciones y derechos.

En un segundo nivel menos abstracto, en las ideologías de los grupos dirigentes se encuentran representaciones sistemáticas que tienen intenciones políticas y objetivos estratégicos. Defino a los grupos dirigentes como aquellos que tienen un mayor control relativo -en relación con otros grupos sociales-de las reglas del juego social y político que abarca o afecta a toda la sociedad, al mismo tiempo que cuentan con la capacidad de conseguir los recursos que demandan sus necesidades básicas estructurales (seguridad, riqueza, consenso, sentido, popularidad, etc.). Por contraposición, los grupos dirigidos son aquellos grupos que tienen menos control relativo sobre las reglas del juego y sobre los recursos que gratificarían ciertas necesidades básicas propias y de otros agentes sociales. En las sociedades democráticas, el recurso que tienen estos grupos es cierta capacidad para expresar su disenso o su consenso con respecto a una determinada política, persona o decisión.

Los grupos dirigentes desarrollan cierta concepción de lo que debería ser una agencia con autonomía. La agencia puede ser el individuo, la clase social, la organización (empresa, la Iglesia, el partido, la secta, el grupo político, el movimiento social o el Estado). Y de acuerdo con este ideal de la agencia social desarrollan un proceso de institucionalización de la autonomización de este agente y de la desautonomización de otros agentes (p. e., se pasa de una concepción que desvincula la cantidad de los aspectos que pueden ser controlados por el Estado y que pasan a estar dentro de la autonomía del individuo). Entonces, los grupos sociales sintetizan el conocimiento que adquieren de un modo más inconsciente (las metanarrativas) y lo que tienen en su conciencia discursiva (Giddens, 1995a) de tal manera que terminan por clasificar la realidad social de un modo más o menos eficaz. Por medio de procesos de institucionalización diferencian, vinculan, producen analogías o separan aspectos de la realidad que estaban unidos (Douglas, 1996: 124 y ss.). 
Estos grupos sociales proyectan el tipo de agente que consideran legítimo de acuerdo con una imagen de ser humano ideal que tienen en su concepción ideológica. Esta imagen ideal no es necesariamente la que manifiestan en el escenario público. Por el contrario, suele ser el resultado de la combinación de intereses reconocidos, por un lado, e intereses no reconocidos de un modo público, por otro. Los intereses reconocidos se encuentran definidos dentro de su discurso de formación de identidad, mientras que los intereses no reconocidos se pueden inferir de sus comportamientos objetivos.

Los grupos burgueses que comenzaron protagonizando los movimientos de protesta en Europa a comienzos del siglo XIII y XIV, terminaron con un discurso público organizado alrededor de los valores de igualdad, libertad y fraternidad tres siglos después. Esta mentalidad burguesa, es decir, mercantilista y capitalista, individualista en relación con el Antiguo Régimen, racionalista, realista o pragmática, fue transformándose hasta la formación de un Estado que tuvo necesidad de definir intereses diferentes a los que proclamaban estos tres valores revolucionarios (Romero, 1967; Elias, 1996).

Por otro lado, los grupos proletarios a los que Karl Marx (Gemkow, 1968) dirigía sus artículos periodísticos y acerca de los que pensaba que no tenían suficiente conciencia de grupo o de clase, sin embargo, mantenían ciertas ideas y un conocimiento de la realidad que habían heredado de la filosofía de los autores burgueses (Kant, Schiller, Schelling, Hegel, etc.). Marx debió luchar en el campo intelectual para desbancar a estos autores porque el conocimiento producido por ellos generaba consecuencias sobre los grupos proletarios más allá de su refutación. Y mucho más tarde, incluso cuando comenzaron a tener conciencia de grupo, el proletariado podría continuar pensando la realidad desde el punto de vista de concepciones más tradicionales. El ejemplo más claro y terrible al mismo tiempo es el de los campesinos rusos perseguidos por Stalin (Howbsbam, 1998: 380 y ss.).

\subsection{Institucionalización de los agentes: proceso de autonomización o desautonomización de ciertos agentes} sociales ¿Cómo se forma y reforma la identidad de los agentes sociales?

¿Por qué los grupos sociales que dirigen o que son dirigidos tratan de definir de un modo histórico las agencias relevantes en cada sociedad? La definición de lo qué es una agencia para cierto grupo social depende de qué imagen ideal de ser humano tiene -de su teoría antropológica-y de lo que pretende conseguir en una coyuntura social determinado grupo social.

El proceso de autonomización y desautonomización social es lo que termina por construir socialmente y por hacer real a una agencia humana. Las sociedades humanas en sus formas históricas definen cuáles serán los agencias preponderantes en su sociedad: la persona individual, una zona o dimensión del individuo (lo 
emocional o lo racional), un grupo social (clan, familia, gremio, etc.), una clase social, un tipo de organización (la empresa, por ejemplo) o el Estado como instituto de regulación de la sociedad como un todo.

La autonomía que tienen los agentes dentro de la sociedad es el nivel de alcance que tiene un determinado agente -persona, grupo, organización, sistema social, sociedad - para hacer algo con independencia de otros. Autonomía debería definirse como el nivel de control que un agente podría y debería tener sobre aquello que se considera parte de su identidad y lo que lo define por lo que es. Agencia es aquella entidad social con capacidad de decidir sobre sus propias acciones. Para hacerlo necesita cierta capacidad de intervención y de reflexividad. En definitiva, la autonomía que tiene algo es lo que lo convierte en un agente. El individualismo, el colectivismo, el personalismo o el socialismo son concepciones que forman imágenes ideales del ser humano y de su vida por medio de relatos, metarrelatos o "formaciones discursivas" (Foucault, 1970: 50-64) que, por medio de instituciones históricamente situadas y de ideologías, definen los procesos de agenciación humana. Este proceso de agenciación se realiza por medio de la atribución de ciertos atributos que conceden mayor grado de autocontrol y de autonomía a ciertos agentes.

Las formas de socialización -en el mismo sentido que Georg Simmel le dio al término (1986)- que permiten que se modifiquen los procesos de autonomización funcionan por medio de mecanismos sociales y psicológicos que se engloban en los denominados procesos de civilización (Elias, 1993: 17 y ss.). Estos procesos de autonomización son los procesos de socialización por medio de los que se instituye un cierto tipo de agencia social en un determinado momento histórico. Es una forma de conocimiento social que se encuentra tanto en las metanarrativas como en las ideologías. Esto significa que es posible que dos grupos sociales que compiten por el poder de una coyuntura crean y defiendan y clasifiquen el mismo tipo de agencia humana. La autonomización concede mayor responsabilidad a un cierto agente, es decir, más capacidad de decisión y más independencia sobre las acciones, los pensamientos, los objetos, los espacios, los tiempos y las emociones de las que tenía antes o en relación a otros agentes.

Desde un punto de vista de la historia de la ciencia, los dos conceptos de agencia más conocida son el individuo y la sociedad como colectivo. Las diferentes formas de individualismo, ya sea en su versión ontológica o metodológica, consideran que la agencia humana por excelencia es el individuo. La única entidad o realidad social con capacidad de decidir y de actuar con responsabilidad es el individuo. Cualquier otra realidad social (grupos y organizaciones) no son reales (individualismo ontológico) o no tienen la capacidad de modificar la realidad social (individualismo metodológico). El individuo puede ser una agencia emocional o racional, puede ser el resultado de una combinación de ambas o puede estar representado y definido por algún hecho institucional (religioso, militar, empresario, etc.). 
La otra concepción de agencia humana es la del colectivismo. En este caso, no son los individuos sino todas las formaciones sociales constituidas y construidas por relaciones y combinaciones de individuos lo que se transforma en lo único real (colectivismo ontológico) y en lo que tiene capacidad de cambiar a la sociedad (colectivismo metodológico). Los individuos que forman los grupos o las organizaciones pierden su condición individual o deja de ser importante su singularidad y, por el contrario, comienza a ser relevante el hecho de que están en un entramado de relaciones que los controla y los domina. Por ende, la agencia humana es trasladada al grupo (grupo étnico, clase social, grupo profesional, grupo de parentesco, organización, etc.).

Lo que esto significa, dentro del problema que se ha planteado antes, es que cualquier transformación de lo privado sobre lo público o viceversa, se puede observar en el proceso de cambio al concederle mayor autonomía o menor autonomía a las personas individuales o a los grupos sociales. En realidad, la diferencia entre lo privado y lo público se encuentra en la diferencia entre el colectivismo y el individualismo ontológico y metodológico.

El proceso de autonomización de cierta agencia implica que ésta tenga más independencia relativa para actuar. Cuánta mayor autonomía se tiene, menos son las "consecuencias indirectas" (Dewey, 1991: 48 y ss.) que se pueden ocasionar con sus acciones. El hecho de que un agente tenga el máximo de autonomía significa que nadie puede considerarse afectado por lo que él hace: es decir, sus acciones no producen efectos indirectos. Dewey diferencia entre las "consecuencias directas" de las acciones -con efectos sobre alguien que se encuentra presente mientras el otro produce la acción-y las "consecuencias indirectas" de las acciones. Éstas últimas son las "acciones públicas" porque afectan a personas o a grupos de personas que no se encuentran expuestos directamente a la acción del otro. Si perciben e interpretan que han sido afectados por las acciones de otros, significa que la acción ha sido institucionalizada como una acción pública.

Esto significa que la persona que ha producido la acción superó su círculo de autonomía relativa. La autonomía es una construcción social, cooperativa e institucionalmente definida. Cuanta más autonomía de un agente, más se encuentra en el ámbito privado. Cuánto menos autonomía tiene, más cantidad de acciones de consecuencias indirectas produce.

$\begin{array}{llll}\text { Consecuencias indirectas } & = & \text { acción pública }== & \text { menos autonomía } \\ \text { Consecuencias directas } & = & \text { acción privada }== & \text { más autonomía }\end{array}$

La autonomía significa mayor independencia y menos procesos de interdependencia o de dependencia. Que no sea posible que otros lo determinen y que tanga capacidad de autodeterminación es lo que concede a alguien la característica de autonomía, principal atributo de la agencia. 
Rom Harré (1982) tiene un concepto sustancialista de agencia humana. Él cree que el agente humano es la persona individual. Pero más allá de esta concepción particular de agente, avanza hacia la definición de las características generales con las que debería contar un agente, más allá de que sea una persona o un grupo de personas. Estas características son:

(a) Autonomía relativa, es decir, que la conducta (acciones y actos) no esté determinada de una manera compulsiva por el ambiente inmediato (Harré, 1982: 257).

(b) Capacidad de representación como una condición para alcanzar autonomía. La representación es la capacidad de imaginar, de forma pública o privada, ciertos futuros posibles (Harré, 1982: 257).

(c) Capacidad de realización como una manera de decidir hacer o de no hacer algo orientado a los futuros posibles que se conocen gracias a la capacidad de representación (Harré, 1982: 257).

(d) Reflexividad limitada para auto-conocerse a sí mismo y para autodeterminarse a actuar (Harré, 1982: 258).

(e) Autointervención o capacidad para actuar sobre las reglas, los planes, impulsos y sentimientos que se experimentan (Harré, 1982: 258).

Como se dijo antes, Harré considera que estos rasgos están de modo exclusivo en las personas. Sin embargo, es posible que estos rasgos sean considerados por ciertos relatos o ideologías como constitutivos de grupos completos o de organizaciones. El proceso que se denomina construcción social de la realidad (Schelsky, 1967; Berger y Luckmann, 1989; Searle, 1997) permite definir a un determinado agente con estas características.

El análisis de Margaret Somers (1995) sobre las bases del concepto de "espacio público" de Habermas y de "cultura política" de Parsons parte de la hipótesis de que son el resultado de la teoría anglo americana del ciudadano. Si bien puede ser real esta aproximación, Somers no tiene en cuenta que hay un proceso más general que es el de la autonomización. La autonomía es un atributo de lo privado para la autora (Somers, 1995: 257). Sin embargo, cuando se considera que el Estado es una agencia, el Estado tiene tanta autonomía como el individuo. No sólo se encuentra esta definición de agencia en el discurso de los revolucionarios de la burguesía, sino que es fácil encontrarlos en el discurso socialista, en el discurso comunista o en el nacionalista.

Identificar cuál es la definición de ser humano ideal en la ideología de un determinado grupo social con cierta posición de poder relativa permitiría inferir los aspectos que el ser humano puede tener bajo su control y cuáles aspectos controla un agente que no es la unidad mínima de ser humano. No es necesario que 
esta imagen ideal de ser humano sea consciente en cada caso. Ni siquiera los científicos suelen contar con una imagen de ser humano construida o definida de modo consciente (Mills, 1997). Mucho más difícil lo es dentro de teorías precientíficas de los sujetos conocidos (Vasilachis de Gialdino, 2003: 19-48). La definición de agencia humana de Harré podría ser aplicada a concepciones individualistas, personalistas, relacionalistas y colectivistas. Un movimiento político (por ejemplo el peronismo) o una clase social (el proletariado) han sido presentados y definidos en los discursos político y científico con capacidades y característica de agencia porque:

(a) Tienen mayor autonomía relativa que otros agentes porque pueden decidir sin ser determinados necesariamente por éstos y porque tienen capacidad de actuar sin que éstos se puedan sentir afectados;

(b) Cuentan con una mayor o menor capacidad de representarse el futuro (Marx explicó con claridad por qué el proletariado no podía percibir el presente y el futuro de un modo más realista a como lo hacía);

(c) cuentan con cierta capacidad de realización o de acción como resultado de la representación que hacen del presente y del futuro;

(d) utilizan la capacidad de pensar en sí mismos o de reflexionar sobre la base de metodologías de discusión, votación, deliberación y negociación (Elster); los grupos que cumplen funciones por delegación, analizan situaciones en representación del resto de los miembros y toman decisiones que el resto aceptará;

(e) finalmente, pueden autointervenirse para cambiar o para modificar algún rasgo que consideran que es negativo de la identidad social del grupo, de sus metas o de sus acciones.

Alejandro Llano (1996: 209) se refiere a este problema como el problema del bien común: esto es el punto intermedio y justo entre los beneficios para la comunidad sin perjudicar a la persona, es decir, sin perder de vista que las personas no se pueden perjudicar o sufrir en lo subjetivo existencial. Este es un punto medio al cual es muy difícil llegar: la autonomía se distribuye entre la persona y cualquier forma de comunidad relevante, pero partiendo de la concepción de persona humana. Llano, filósofo español de tradición realista y metafísica, intenta hacer una consideración universal sobre el problema de la moral pública en la que no puede dejar de lado a la persona humana individual. Sin evaluar hasta dónde uno puede coincidir con su punto de vista, lo cierto es que es un ejemplo del modo en que las teorías definen cuál es el agente con capacidad de decisión más importante. Llano no puede dejar de lado a la persona porque considera que es ella la que representa el agente por excelencia del mundo humano. 
Pueden existir contradicciones entre la concepción ideológica de un grupo y el modo de actuar en la praxis social. Muchas veces, las teorías que tienen consecuencias sobre la realidad social, presentan contradicciones porque sus objetivos y metas no llegan a hacer reales de un modo coherente con los principios y valores fundamentales.

\subsection{Institucionalización de lo que aparece y de lo que no aparece: teoría política y teoría social de la publicidad}

La segunda función directa de la diferencia entre lo privado y lo público está relacionado con lo que se puede y se debe mostrar y aquello que no se puede o no se debe mostrar a otros. El hecho de que se pueda o de que se deba mostrar o no mostrar algo está determinado por aquello que molesta o que incomoda, que se considera inmoral o malo, que no se cree normal, etc. Es un hecho corroborado por la antropología social y la sociología que todas las sociedades humanas, de cualquier época, dividen su vida social en dos zonas o modos de comportarse: una en la cual es legítimo y válido, normal y bueno mostrarse ante los sentidos y la percepción de otros miembros de la sociedad; otra zona, en cambio, que se usa para que las personas oculten comportamientos, ideas, objetos, etc. Algunas de las categorías socioepistemológicas que dividen lo que se oculta y lo que se muestra son, entre otras: normal/anormal, bueno/malo, alto/bajo, central/periférico, legítimo/ilegítimo.

La definición de agente y la autonomía de este agente está acompañada por el proceso de institucionalización de lo que ese agente puede, debe y tiene capacidad de mostrar a otros que están fuera de su círculo de control o de autonomía. Aquello que se puede mostrar o lo que no se puede mostrar está determinado por representaciones morales, técnicas y cognoscitivas. Lo que se denomina privado y público, entonces, es la diferencia entre lo que tiene más o menos autonomía, por un lado, y lo que puede o no puede ser mostrado o publicitado por otro.

La autonomía o el hecho de ser considerado una agencia social definen unos límites de identidad para la agencia humana que lleva a que se definan, casi de un modo natural -aunque en realidad es un modo naturalizado - lo que puede y lo que debe mostrarse y/o ocultarse. Por ejemplo, en las sociedades modernas el Estado es una agencia. Pero hay diferencias entre la autonomización del Estado entre sociedades con políticas democráticas y sociedades con políticas autocráticas. En un Estado democrático, un ciudadano puede demandar al Estado. Esto significa que el Estado no tiene autonomía absoluta. Y para restarle autonomía se instituyeron procesos de publicitación de las decisiones de los diferentes poderes, mayor visibilidad y exposición de sus funcionarios (Thompson, 2001). 


\subsubsection{La fenomenología de la aparición}

Hannah Arendt (2002: 15 y ss.) considera que la vida del espíritu, del pensamiento, comienza con el proceso de captación y percepción de lo que aparece por parte de un sujeto que conoce. No se puede creer en que algo existe si no hay indicios, signos o síntomas que declaren la presencia directa, deformada o escondida de algo real.

Arendt considera que las funciones radicales y constitutivas de la vida pública o de la "esfera pública" como ella la denomina, son dos. En primer lugar, el hecho de que todos puedan ver y oír algo que sucede aumenta el efecto de realidad de lo que ha sucedido. Con lo cual, algo que es real, aumenta su efecto de realidad si es observado o percibido por todos. En segundo lugar, la otra función antropológica de la esfera pública es generar un efecto de continuidad que combate la futilidad y lo pasajero de la vida privada (Arendt, 1993: 59-67).

\subsubsection{Teoría social de la publicidad}

La teoría social de lo que se debe, se puede o no se puede mostrar, se encuentra explicada en la teoría de la civilización de Norbert Elias (1993) y en el análisis que Anthony Giddens (1995a) hace de la intimidad en años actuales. En primer lugar, el pudor es un mecanismo de control universal, es decir, un mecanismo que afecta a la especie. Permite que las personas controlen ciertas acciones, ciertas palabras o frases. Esconder o mostrar determinadas partes del cuerpo, ciertos objetos y espacios puede depender -y de hecho dependedel canon social que se denomina pudor. Sucede que se moraliza lo que se puede o no puede, se debe o no se debe mostrar y esconder para que las personas desarrollen vergüenza de hacerlo (Kaufman, 1994: 91 y ss.; Arendt, 1993: 78). Los procesos de aparición o de no aparición presentan situaciones contradictorias. Los grupos dirigentes que están en relaciones conflictivas pueden tener diferentes representaciones de lo que debería aparecer y no aparecer. Segundo, los grupos no dirigentes suelen expresarse y manifestar su disenso sobre las acciones de los grupos dirigentes por medio de la publicidad de situaciones, objetos, acciones, personas, ideas, etc. que están en contra del proceso mismo de autonomización construido por los grupos dirigentes. Lo que se oculta y lo que se muestra en una sociedad está determinado por aquello que se ha considerado agencia y por el modo en que se construye la identidad de esta agencia. Algunas de las categorías que clasifican mejor el material que luego se mostrará o que se esconderá.

\subsubsection{Lo normal y lo anormal}

Alfred Schütz es el maestro de la definición de las maneras de diferenciar lo normal de lo anormal. Los seres humanos vivimos dentro de "mundos" culturales considerados normales por los que se encuentran "dentro" y menos normales por aquellos que se consideran y están "fuera" de esos mundos. Lo que es normal, 
entre otras cosas, se caracteriza por aquello que aparece ante todos sin generar problemas, es decir, algo que aparece de un modo aproblemático (Schütz y Luckmann, 1977). .

Una de las principales categorías que clasifican aquello que puede mostrarse en el límite que trasciende a la identidad de la agencia es la de la diferencia entre lo normal y lo anormal. Lo normal es lo que aparece sin producir conmoción en las personas sociales. Algo que es necesario que sea considerado "normal" debe, antes que nada, aparecer como real, estar presente, desocultarse de la percepción de los contemporáneos. Por eso, en cuanto alguien pretende que algo se convierta en normal, debe hacerlo aparecer en el escenario público.

\subsubsection{Lo bueno y lo malo}

Dentro de lo considerado normal y anormal puede clasificarse la realidad como "buena" o "mala" desde un punto de vista moral. Hay aspectos de la realidad que se consideran normales que pueden ser clasificados, además, como "malos" o como "buenos". Desde este punto de vista, se puede mostrar lo que es malo o lo que es bueno mientras que es normal. El problema de lo que es bueno o malo adquiere mayor intensidad en el caso de que sea anormal. Cuando aparece algo anormal y malo en el escenario público se produce un "escándalo genuino" ${ }^{1}$, es decir, una conmoción real producto de la vergüenza social por percibir el acontecimiento. En cambio, hay "escándalos no genuinos" que son las conmociones exageradas por los agentes sociales al conmocionarse por algo que consideran malo, pero normal. Entre este grupo, los escándalos cumplen funciones políticas y éticas de control y de sanción social. Las sociedades pueden mantener este tipo de diferencia por mucho tiempo.

El análisis de Eduard Fuchs (1996) de la moral sexual en la época burguesa muestra con claridad el modo en que se combinaba la doble articulación de clasificaciones: bueno/malo, normal/anormal. Frente al discurso público de los grupos dirigentes y de las personas de la clases con más alto rango o posición social, los periódicos a partir de 1860 comenzaron a mostrar algunos aspectos de la sociedad, que quedaban en la zona de la vida privada, pero que existían: prostitución de menores, avisos de citas de personas casadas, solicitud de casamiento con personas de fortuna, etc. Todo esto existía antes de que fuera mostrado y expuesto: era normal para muchos contemporáneos. Para estos, el escándalo sólo era una representación o una ac-

\footnotetext{
1 No estoy seguro de que esta diferencia la mantenga John Thompson (2001) en su estudio monográfico sobre los escándalos. Sin embargo, creo que la distinción no es menor. No es lo mismo que mientras el "rey se pasea desnudo" aquellos que lo ven no digan nada, a la situación en la que nadie puede ver si el rey está desnudo o vestido, cosa que se aclara después de algún acontecimiento inesperado. En el primer caso, el escándalo existe pero no es genuino ya que la opinión pública conoce la verdad acerca del hecho. En el segundo caso, en cambio, el escándalo es genuino porque la trasgresión de los valores se descubrió después de un proceso de encubrimiento y de mentiras.
} 
tuación pública, pero no llegó a ser un escándalo genuino. Tanto una como otra clasificación han sido producidas por el proceso civilizatorio (Elias, 1993) ya que, este proceso, si no alcanza a moralizar de un modo eficaz a las personas sociales, por lo menos -como lo muestra el trabajo de Fuchs - separa de un modo contundente entre la zona privada y la zona pública de la conducta. Este resultado se obtiene por medio del desarrollo de la vergüenza social, del sentimiento de culpa y de sensaciones de asco o de formalidad ante ciertas situaciones humanas o no humanas.

\subsubsection{Lo alto y lo bajo}

La clasificación que las instituciones humanas realizan gracias a los metarrelatos o a las representaciones de los grupos sociales de "lo-que-está-más-arriba" (y por ende es mejor) y "lo-que-se-encuentra-más-abajo" (y es peor) desde un punto de vista social, queda articulado con las dos formas anteriores de clasificación. Las diferencias sociales, las distinciones de posición y de estamento social condicionan a las categorías bueno/malo, normal/ anormal. Algo es bueno o malo, normal o anormal, sólo si es posible "quién" es el que lo hizo. El "quién" se define por medio de su identidad social, es decir, gracias al lugar que ocupa en la estructura social general.

\subsubsection{Lo importante y lo accidental, lo central y lo periférico}

Por lo general, lo que se considera "importante" (normal/anormal, bueno/malo, alto/bajo) puede ser que genere una mayor tensión social a la hora de decidir si se puede o si se debe o no mostrarlo y/o esconderlo de la percepción de otros. Lo que una sociedad ha ubicado en un lugar poco relevante, es decir, aquello que es "accidental" para el entramado institucional de una determinada sociedad, no genera, por el contrario, problemas o conflictos acerca de la necesidad, deber o poder para mostrarlo y/o esconderlo.

\subsubsection{Lo legítimo y lo ilegítimo}

Cualquiera de los otros modos de diferenciar las conductas sociales que aparecen o que se esconden a la observación y a la percepción de los demás se organiza dentro de las categorías de lo legítimo y de lo ilegítimo. Las estrategias de aparición en el escenario público están pensadas para legitimar o para invalidar la acción, el discurso, una expresión, una explicación o un debate entre agentes. ¿En qué medida lo normal o lo anormal, lo bueno o lo malo, lo importante o lo periférico, lo alto o lo bajo son enmarcados en un contexto de legitimación o de deslegitimación?

En definitiva, las diferencias entre lo que se muestra y se esconde están relacionadas con el nivel de autonomización que se acepta en una sociedad. Si se acepta que es el individuo el agente que debe tener mayor nivel 
de autonomía relativa en comparación a otros, es coherente que la publicidad normal y corriente realizada en la sociedad esté relacionada con los aspectos y objetos que dan y construyen la identidad de este agente.

\subsubsection{Teoría política de la publicidad}

La teoría política de la democracia representativa es la contra-cara de la teoría del arcana imperii (Bobbio, 1989; Schmitt, 1990: 67 y ss.). La necesidad de que el señor o el príncipe mantengan en secreto sus decisiones o no justifique en ningún momento por qué actúan del modo en que lo hacen, depende de una concepción acerca del gobierno eficaz.

La democracia moderna introdujo el mecanismo de la publicidad para controlar a los representantes del pueblo y para regenerar el pensamiento de éste. Las relaciones entre los gobernados y los gobernantes en las democracias representativas se definen por medio de este mecanismo de exposición ya que los gobernantes están dirigiendo algo sobre lo que no tienen autonomía. Lo que gestionan y sobre lo que trabajan no es parte de su condición de políticos. Deben mostrarlo y estar dispuestos a ser observados.

En síntesis, el proceso de mostrar o de no ocultar comienza con las raíces psíquicas y sociales de comportamientos básicos de los seres humanos y termina en el ordenamiento de la política. Es decir, comienza como dimensión prepolítica y se transforma en una parte central de la política: la teoría social de lo que se expone y se esconde antecede a la teoría política, aunque también ésta puede determinar el modo en que la sociedad se comporta en público y en privado.

\subsection{Aspectos, elementos y objetos sociales sobre los que se realiza la "autonomización" y la "publicidad"}

Los aspectos de las personas y de los grupos, los temas y los objetos sociales que permanecen dentro de la zona de autonomía del agente en cuestión y que se publicitarán o se esconderán están relacionados con la identidad social con que se define y se identifica el agente.

En el Antiguo Régimen, las personas debían y necesitaban participar en rituales públicos de la religión católica ya que la vida social se definía por medio de la vida religiosa, mucho más que en el siglo XX. Pero además, las interpretaciones acerca de lo que la gente hacía bien o mal no sólo tenía efectos sobre la misma persona que pecaba, sino sobre la comunidad. Al ampliar la zona de responsabilidad sobre la comunidad en lugar de centrarla en el individuo, los objetos sociales que se deben exponer o que se deben esconder dependen de esta concepción de la identidad del ser humano (Verdo, 1998: 225-228).

La sociedad moderna definió que lo que debía aparecer legitimado eran los resultados de las decisiones racionales de las personas públicas o del Estado. Las acciones emocionales debían quedar dentro de la vida 
privada. Las emociones no sólo eran objetos negativos sino que también podían quedar dentro de la autonomía de la persona individual si no eran mostrados delante de otros.

Si bien se podría considerar que era normal que una persona experimentara emociones fuertes, no se evaluaba como bueno que las expusiera a los otros. Las personas que pretendían mostrarse como superiores a otras, debían tener una capacidad de control de las emociones que debía ser mayor a la de las personas inferiores en status social. Por otro lado, las emociones eran categorizadas como poco importantes. No era central preocuparse por las emociones. Pero, ¿Cuándo se puede decir que era legítimo salirse de los límites de lo pautado como las fronteras de lo público y de lo privado? Las manifestaciones emocionales podían expresarse en público de un modo legítimo dentro de una actividad deportiva, por ejemplo.

2.5. Espacios y tiempos sociales en los que se muestran o esconden los aspectos y objetos sociales (medios de comunicación, tribunales, espacios de la ciudad, universidad, escuela, consejo deliberante y parlamentos, etc.)

El modo de ejercer efectos sobre la realidad social es que los procesos de autonomización y de publicitación se encuentren en ciertos espacios sociales. Los espacios sociales tienen diferentes características. Pero comparten una: son sitios en los que pueden concurrir personas con posiciones y funciones diferentes en la sociedad.

En primer lugar, los medios de comunicación de masas son espacios sociales centrales en las sociedades contemporáneas. Esto se debe al gran número de personas que se exponen a ellos. La cantidad es una categoría relevante en este espacio. Además, estos medios definen, a mediano plazo, las condiciones cognitivas desde las cuales pensar no sólo la realidad mediática: estos medios transforman en naturales los modos de pensar y de clasificar la realidad en general. Finalmente, los medios definen ciertos criterios de diferenciación entre lo privado y lo público con mucha más contundencia que otros actores o agentes.

Segundo, hay otros espacios en los que se exponen los objetos seleccionados, con cierto grado de apertura, por unos agentes. Su relevancia, en cambio, se encuentra no en la cantidad de personas sobre la que influyen sino en la calidad o cualidad de las personas y en su grado de decisión. Los tribunales y los parlamentos son dos espacios sociales importantes en las sociedades actuales.

Tercero, los ámbitos de exposición cultural (universidades, salas de conferencia, clases, salas para muestras de arte, salas de lectura, biblioteca, etc.) son otros espacios que permiten que los agentes autonomizados expongan o hagan publicidad de lo que tienen que hacer.

En cuarto lugar, un espacio central es el de la ciudad. La aparición de ciertos agentes y de sus propuestas en el ámbito de la ciudad obtiene la atención de una gran audiencia.

$146 \mid n^{\circ} 7$ | doxa.comunicación 
En general, estos espacios no sólo son sitios de aparición sino que funcionan como frames (Minsky, 1986; Goffman, 1986; Bateson, 1991a, 1991b) ya que cada uno de ellos define patrones subyacentes que ayudan a dar sentido y a entender lo que sucede en esos espacios.

2.6. Actores que encarnan las acciones públicas y privadas en cada espacio de acuerdo con los objetos sociales que deben controlar o que deben publicitar

El escenario público es una metáfora que se considera más representativa y realista que la de "espacio público" (Habermas, 1986) o la de "esfera pública" (Arendt, 1993). Aquello que cuenta con la necesaria y suficiente autonomía como para ser considerado una agencia humana actúa en el escenario público siguiendo un guión que está definido y socializado por las metanarrativas (Somers) que delimitan la organización política de las sociedades y los límites y alcances de los Estados. En realidad, en las sociedades del capitalismo avanzado del siglo XX, la vida pública estuvo dividida entre aquello que el Estado considera que es parte de su autonomía y que, en consecuencia, debe controlar, por un lado, y, por otro, lo que el Estado debe mostrar para transparentarse ante la sociedad civil y la opinión pública, es decir, los ciudadanos; pero los agentes privados también afectan lo público ya que sus acciones terminan produciendo consecuencias, de hecho, sobre el resto de los grupos sociales. Aquello que los agentes de la sociedad civil deben hacer podría explicarse dentro de un marco de acción que es análogo a un escenario. Un escenario en el que los agentes con más o menos autonomía tienen cierta capacidad de acción, de discurso, de negociación, de aparición, de habla, de comportamiento. El Estado, las empresas, los sindicatos, los movimientos sociales, las organizaciones sin fines de lucro, las empresas y los medios de comunicación son agentes que se transforman en actores para hacer y desarrollar toda esa gama de comportamientos.

Las relaciones sociales entre los grupos dirigentes y dirigidos terminan por institucionalizar los siguientes aspectos estructurales del escenario público: primero, las diferencias entre los agentes con mayor o menor autonomía (continuum privado-público), segundo, los criterios para diferenciar cómo se debe mostrar o no mostrar; tercero, los objetos sociales que están dentro y fuera de la autonomía del agente y que también son parte de la identidad de éste. Por eso, se seleccionan los objetos y los aspectos que se muestran y que no se muestran de cada agente por medio de la acción y del desempeño en los espacios sociales definidos por los grupos dirigentes o no dirigentes.

Los agentes transformados en agencia humana por el proceso de autonomización y de ocultación y exposición, se transforman en actores, es decir, adquieren cierta capacidad de dramatizar o de participar en situaciones que pueden ser percibidas por otros al realizarse en el escenario público. 
Los agentes se transforman en actores, es decir, en agentes con capacidad de actuar y de representar una imagen subjetiva, mostrar intenciones y construir cierta reputación; puede ser que estos actores desarrollen varias funciones diferentes: (a) expresan intenciones y estados internos (p.e., el enojo o el malestar por lo que alguien ha hecho), (b) se relacionan con otros (p. e., piden ayuda, tratan de buscar alianzas, se distancian, critican a otros, etc.); (c) hacen referencia o describen cierta realidad objetiva (p. e., exponen los beneficios de un plan de gobierno o explican cómo sucedió un accidente); (d) discuten con otros actores acerca de cualquiera de los otros puntos (a, b y c) (Bühler, 1966: 47 y ss.; Habermas, 1990: 67 y ss.)

2.7. Principios de regulación de lo que hacen los agentes o las pautas de comportamiento y las reglas del escenario público

Los actores se relacionan con otros agentes de acuerdo con ciertos principios de regulación (Habermas, 1986: 66) o de ordenamiento de la aparición pública. Para qué y por qué actuar en público es la pregunta que se debe responder para entender los principios de regulación. Se puede actuar para conseguir reputación, para conseguir fama o prestigio, para adquirir notoriedad, para discutir, para ser familiar, etc.

La estructura del escenario público está compuesto por los agentes que participan -transformándose en actores - y por sus relaciones sociales. Las pautas de comportamiento son el modo habitual de comportarse que muestran estos agentes en su función de actores. El desarrollo de las pautas, es decir, qué es lo que hacen los actores en el escenario público y qué es lo que deberían hacer (discutir, representar a alguien o a algo, adquirir fama, adquirir o perder reputación, honor, prestigio, ser notorios, ser populares, etc.) condiciona los procesos políticos de representación y de inclusión social. La representación está condicionada por los modelos de democracia indirecta en el modo en que los ciudadanos pueden controlar a sus representantes por medio de la publicidad de sus decisiones o propuestas. La inclusión social debería estar definida también como la inclusión que los actores o los agentes tienen en los procesos de visibilidad en el escenario público, sea para ser controlados o para que adquieran una existencia cargada de sentido que se manifiesta en el hecho de conocer cómo es alguien y qué es lo que le sucede en su vida. Desde la imagen escéptica que Habermas dejó con su análisis del espacio público burgués, se continúa con el intento por encontrar un lugar en el que se puedan desarrollar sin restricciones las acciones discursivas y no discursivas de los actores sociales que tienen mayor o menor autonomía como agencias.

\subsubsection{Aparecer para expresar o esconder algo interno}

Aunque esto parezca ser un objetivo poco realista, sin embargo, es uno de los mecanismos más importantes de manipulación o de comunicación en el escenario público (Durandin, 1995; Schelling, 1982). La 
aclaración o el mantenimiento oculto de las intenciones que tienen los actores es uno de los modos más eficaces para alcanzar objetivos en el escenario público. Si las audiencias conociesen las intenciones no formales y públicamente declamadas de los actores, podrían inferir mucha más información relevante para entender lo que sucede en el sistema político.

\subsubsection{Establecer una relación con alguien}

Actuar y hablar en el escenario público suele hacerse para establecer relaciones de alianzas, compromisos, amenazas o falsas evidencias para engañar.

\subsubsection{Instalar un discurso acerca de algo}

Algunas veces sólo se pretende decir algo sobre algo. Pero esto siempre implica una posición en relación con alguien o con otros actores.

\subsubsection{Discutir con alguien}

Intercambiar argumentos con otro actor implica ponerse al descubierto acerca de lo que importa o no importa, lo que es o no es normal, lo que es bueno o malo, etc.

\section{Consideraciones finales y discusión}

La dicotomía entre lo "público" y lo "privado" se constituye y se origina en la manera en que se organiza el "escenario público". Desde el punto de vista de la sociología de la comunicación, el escenario público, tal como se ha presentando antes, sintetiza y materializa las dimensiones que constituyen y realizan a estas dos zonas de vida social. El escenario público se compone de los espacios y de los tiempos sociales de aparición y de ocultamiento de los actores, que se desempeñan en él gracias a ciertas pautas de comportamiento institucionalizadas que definen y precisan continuamente el grado de autonomización y de desautonomización de las agencias humanas. Estos componentes estructurales del escenario público modifican los límites de las dos zonas de acción social. De esta manera, lo público y lo privado son criterios sociales por medio de los que las sociedades históricas diferencian y clasifican el comportamiento de las personas y de los grupos. Y gracias a este concepto de escenario público es posible hacer una lectura de los problemas políticos desde el punto de vista de la sociología de la comunicación. En primer lugar, lo público no puede ser separado de lo privado. Este continuum es el resultado del juego de autonomización/desautonomización de ciertos agentes y de sus relaciones sociales. Lo público es lo que relativiza el poder de un agente transformado en agencia y en actor. Como agencia, los aspectos y temas con los que se identifica y que son considerados pú- 
blicos son aspectos y temas sobre los que no tiene autonomía. Sin embargo, eso mismo que le quita autonomía a un agente le provee a otro. El hecho de que un funcionario público no tenga autonomía absoluta es lo que le permite al ciudadano tener un poco más sobre el funcionario. Por otro lado, la autonomía se consigue con la prestación de derechos y con la sanción de obligaciones para otros agentes.

La interdependencia que se crea con las relaciones de autonomía y desautonomización es la base de la organización política de la sociedad, sea para la representación, la gobernabilidad, la inclusión social o la legitimidad.

En segunda instancia, la exposición y el ocultamiento precisan y definen con rasgos más claros el hecho de que un agente sea una agencia humana para cierto metarrelato o ideología. Tener que mostrar, salir del ocultamiento o exponerse, es un proceso de control que ejerce la comunidad política, porque antes es un proceso de observación o no la continuidad y corroboración de la realidad que lleva adelante la comunidad social.

Tercero, el modo en que los actores se comportan en el escenario público depende, entonces, de estos dos principios: los actores pueden representar ciertos actos si cuentan con cierta autonomía y si respetan o trascienden ciertas fronteras que diferencian lo que se puede o no se puede mostrar.

En definitiva, lo que distingue entre formas de vida privada y pública diferentes son los siguientes puntos:

(a) Ciertos procesos de autonomización por medio de mecanismos de limitar y de formar un agente social gracias a establecer el origen de la autonomía de decisión y el alcance de la responsabilidad de una persona individual, de un grupo, de una clase social, etc.

(b) Un cierto modo de mostrar o de esconder, de aparecer o no aparecer ante los otros, condicionado por el proceso de autonomización, pero también por el grado de sensibilidad psíquica y social que se establece como canon, es decir, utilizando la vergüenza como mecanismo de autocontrol y el consenso o el disenso sobre ciertas formas de conductas.

(c) Las pautas de conducta y de pensamiento que se siguen en la zona privada y pública de vida, es decir, qué se hace y cómo se piensa en cada una de estas zonas sociales; por ejemplo, la vida privada es una zona emocional o también debe ser considerada racional; la vida pública debería ser un lugar de discusión o sólo de aparición; cómo se actúa y qué se hace en cada zona social es el resultado de los dos procesos anteriores, pero es importante para definir el modo en que se modificarán el proceso (a) y el proceso (b). 
(d) La identidad del escenario público condiciona los procesos de representación, gobernabilidad y de inclusión social.

\section{Conclusiones}

La autonomización y el ocultamiento-desocultamiento son los procesos constitutivos de la dicotomía privado-público. La función que cumplen es de control social y de producción de las agencias sociales básicas en una sociedad.

Este proceso coloca las bases para que se estructure un escenario público y una zona en bastidores en la cual se organiza y se planifica lo que es posible, factible y necesario que aparezca y no aparezca, se discuta o no se discuta, se haga referencia o se haga silencio. El escenario público es sólo -aunque no lo debería ser-la dimensión visible de lo que sucede en la zona privada de decisión.

Los agentes sociales utilizan el escenario público para cambiar el modo en que otros perciben y organizan la realidad, para modificar lo que otros piensan de ellos o para amenazar a otros agentes sobre el hecho de que pueden decir o hacer algo que los comprometa ante la percepción de otros.

\section{Referencias bibliográficas}

Arendt, H. (1973): Crisis de la República, Madrid: Taurus (edición en inglés, 1969).

Arendt, H. (1993): La condición humana, Barcelona: Paidós (edición en inglés, 1958).

Arendt, H. (2002): La vida del espíritu, Buenos Aires: Paidós (edición en inglés, 1971).

Bateson, G. (1991a): “Una teoría del juego y de la fantasía”, en G. Bateson: Pasos hacia una ecología de la mente. Una aproximación revolucionaria a la autocomprensión del hombre, Buenos Aires: Editorial Planeta-Carlos Lohle (edición en inglés, 1972), pp. 205-222.

Bateson, G. (1991b): “Las categorías lógicas del aprendizaje y de la comunicación”, en G. Bateson: Pasos hacia una ecología de la mente. Una aproximación revolucionaria a la autocomprensión del hombre, Buenos Aires, Editorial Planeta-Carlos Lohle (edición en inglés, 1972), pp. 309-338.

Berger, P. Luckmann, Th. (1989): La construcción social de la realidad, Buenos Aires: Amorrortu editores (edición en inglés, 1968).

Beuchamp, M. (1998): “Comunicación política y empresa. Algunos puntos de vista teóricos”, en G. Gauthier, A. Gosselin y J. Mouchon (comps.), Comunicación y política, Barcelona: Gedisa Editorial (edición en francés, 1995), pp. 276 -293.

Bobbio, N. (1989): Estado, gobierno y sociedad. Por una teoría general de la política, México: Fondo de Cultura Económica. 
Bühler. K. (1966): Crisis de la psicología, Madrid: Ediciones Morata.

Burke, P. (1995): La fabricación de Luis XIV, Madrid: Editorial Nerea.

Dewey, J. (1991): The public and its problems, Ohio: Swallow Press, Ohio: University Press (primera edición, 1927).

Douglas, M. (1996): Cómo piensan las instituciones, Madrid: Alianza Editorial.

Durandin, G. (1995): La información, la desinformación y la realidad, Barcelona: Editorial Paidós (edición en francés, 1993).

Durkheim, E. (1991): Las reglas del método sociológico. México: Premiá editora

Elias, N. (1989): Sobre el tiempo, Madrid: Fondo de Cultura Económica (edición en alemán, 1984).

Elias, N. (1993): El proceso de la civilización. Investigaciones sociogenéticas y psicogenéticas, México: Fondo de Cultura Económica (edición en alemán, 1977).

Elias, N. (1995): Sociología fundamental, Barcelona: Gedisa editorial (edición en alemán, 1975).

Elias, N. (1996): La sociedad cortesana, México: Fondo de Cultura Económica (edición en alemán, 1966).

Elias, N. (1998): “¿L'Espace privé, o Privatraum o espacio privado”?, en La civilización de los padres y otros ensayos, Bogotá: Editorial Norma, pp. 349-366.

Elizalde, L. H. (1998): "Medios, instituciones y cambio institucional. El caso de la neotelevisión", en Revista Estudios sobre el Mensaje Periodístico, núm. 4, pp. 135-160.

Elizalde, L.H. (2006): “La comunicación gubernamental: problemas y soluciones estratégicas”, en L. Elizalde, D. Fernández Pedemonte y M. Riorda, La construcción del consenso. Gestión de la comunicación gubernamental, Buenos Aires: La Crujía, pp. 145-257.

Fleck, L. (1986): La génesis y desarrollo de un hecho científico, Madrid: Editorial Alianza (edición en inglés, 1935).

Foucault, M. (1970): La arqueología del saber, México: Siglo Veintiuno editores.

Fuchs, E. (1996): Historia de la moral sexual. 3. La época burguesa, Madrid: Editorial Alianza (edición en alemán, 1986).

Genkow, H. (1968): Carlos Marx. Biografía completa, Buenos Aires: Editorial Cartago.

Giddens, A. (1995a): La constitución de la sociedad. Bases para una teoría de la estructuración, Buenos Aires: Amorrortu editores (edición en inglés, 1984).

Giddens, A. (1995b): La transformación de la intimidad. Sexualidad, amor y erotismo en las sociedades modernas, Madrid: Cátedra (edición en inglés, 1992).

Glaser, B. Strauss, A. (1967): The discovery of grounded theory. Strategies for cualitative research, New York: Andine Publishing Company. 
Goffman, E. (1986): Frame Analysis An essay on the Organization of Experience, New York: Northeasterm University Press (edition 1976).

Greimas, A. Courtés, J. (1990): Semiótica. Diccionario razonado de la teoría del lenguaje, Madrid: Gredos.

Grosselin, A. (1998): “Introducción. La comunicación política. Cartografía de un campo de investigación y de actividades”, en Gathier, G., Grosselin, A. y Mouchon, J.: Comunicación y política, Barcelona: Gedisa (edición en francés, 1995), pp. 9-31.

Habermas, J. (1986): Historia y crítica de la opinión pública. La transformación estructural de la vida pública, Barcelona: Editorial Gustavo Gili.

Habermas, J. (1990): “Acciones, actos de habla, interacciones linguísticamente mediadas y mundo de la vida”, en J. Habermas: Pensamiento postmetafísico, México: Taurus (edición en alemán, 1988), pp. 67-107.

Habermas, J. (1998): Facticidad y validez. Sobre el derecho y el Estado democrático en términos de teoría del discurso, Madrid: Editorial Trota (edición en alemán, 1992).

Habermas, J. (1999): “Tres modelos normativos de democracia”, en La inclusión del otro. Estudios de teoría política, Barcelona: Editorial Paidós (edición en alemán, 1996).

Hall, E. (1998): La dimensión oculta, México: Siglo XXI editores (edición en inglés, 1966).

Harré, R. (1982): El ser social. Una teoría para la psicología social, Madrid: Alianza (1979).

Heath, R. L. (1997): Strategic Issues management. Organizations and Public Policy Challenges, Thousand Oaks: Sage Publications.

Hobsbawm, E. (1998): Historia del siglo XX, Buenos Aires: Crítica (edición en inglés, 1994).

Kant, I. (1993): “Respuesta a la pregunta: ¿Qué es la Ilustración?”, en AA.VV.: ¿Qué es Ilustración?, Madrid: Editorial Tecnos.

Kaufman, G. (1994): Psicología de la vergüenza. Teoría y tratamiento de sus síndromes, Barcelona: Herder (edición en alemán, 1989).

Lavigne, A. (1998): "La politización de la información del sector público”, en Gauthier, G., Gosselin, A. y Mouchon, J.: Comunicación y política, Barcelona: Gedisa Editorial (edición en francés, 1995), pp. 257-275.

Lyotard, J-F. (1989): La condición postmoderna. Informe sobre el saber, Buenos Aires: Ediciones R.E.I. (edición en francés, 1987).

Llano, A. (1996): “La verdad en la conversación humana. Una consideración al hilo de la polémica entre el liberalismo y comunitarismo”, en Luis Núñez Ladevéze (ed.): Ética pública y moral social, Madrid: Editorial Noesis, pp. 205-222.

Mills, Ch. W. (1997): La imaginación sociológica, México: Fondo de Cultura Económica (edición en inglés, 1959).

Minsky, M. (1986): La sociedad de la mente. La inteligencia humana a la luz de la inteligencia artificial, Buenos Aires: Ediciones Galápagos.

Núñez Ladevéze, L. (2000): La ficción del pacto social, Madrid: Editorial Tecnos. 
Riorda, M. (2006): “Hacia un modelo de comunicación gubernamental para el consenso”, en L. Elizalde, D. Fernández Pedemonte y M. Riorda, La construcción del consenso. Gestión de la comunicación gubernamental, Buenos Aires: La Crujía, pp. 17-142.

Romero, J. L. (1967): La revolución burguesa en el mundo feudal, México: Siglo Veintiuno Editores.

Schelsky, H. (1967): "Acerca de la estabilidad de las instituciones, en especial de las constituciones. Reflexiones antropológico-culturales sobre un tema jurídico-sociológico", en El hombre en la civilización científica y otros ensayos, Buenos Aires: Sur.

Schelling, Th. (1958): “The Strategy of Conflict Prospectus for a Reorientation of Game Theory”, in The Journal of Conflict Resolution, vol. 2 (3), pp. 203-264.

Schelling, Th. (1982): “Establishing Credibility: Strategic Considerations”, en The American Economic Review, vol. 72 (2), Papers and Proceedings of the Ninety-Fourth Annual Meeting Of the American Association, pp. 77-80.

Schmitt, C. (1990): Sobre el parlamentarismo, Madrid: Editorial Tecnos (edición alemana, 1923).

Schütz, A. y Luckmann, Th. (1977): Las estructuras del mundo de la vida, Buenos Aires: Amorrortu editores (edición en inglés, 1973).

Searle, J. (1997): La construcción de la realidad social, Barcelona: Editorial Paidós.

Simmel, G. (1986): Sociología, 2. Estudios sobre las formas de socialización, Madrid: Alianza Editorial (edición en alemán, 1908).

Somers, M. R. (1995): "Narrating and Naturalizing Civil Society and Citizenship Theory: The Place of Political Culture and the Public Sphere”, en Sociological Theory, vol. 13, Issue 3, Nov., 229-274.

Thompson, J. (2001): El escándalo político. Poder y visibilidad en la era de los medios de comunicación, Editorial Paidós: Barcelona (edición en inglés, 2000).

Van Dijk, T. (1995): “Discurse semantics and ideology”, Discurse \& Society, vol. 6 (2).

Vasilachis de Gialdino, I. (1997): La construcción de representaciones sociales. Discurso político y prensa escrita. Un análisis sociológico, jurídico y lingüístico, Barcelona: Gedisa Editorial.

Vasilachis de Gialdino, I. (2003): Pobres, pobreza, identidad y representaciones sociales, Barcelona: Gedisa Editorial.

Verdo, G. (1998): “El escándalo de la risa, o las paradojas de la opinión en el periodo de la emancipación rioplatense”, en Guerra, F.X. y Lempériére, A. (eds.) Los espacio público en Iberoamércia. Ambigüedades y problemas. Siglos XVII-XIX, México: Fondo de Cultura Económica - Centro Francés de Estudios Mexicanos y Centroamericanos, pp. 225-240. 